\title{
Bottom-up sentiment and return predictability of the market portfolio ${ }^{1}$
}

\author{
Jiaqi Guo ${ }^{2}$, Youwei $\mathrm{Li}^{3}$, Min Zheng ${ }^{4}$
}

\begin{abstract}
This paper provides strong evidence that market sentiment measured bottom-up from individual-stock sentiment is negatively related to future long-term market returns and is positively correlated with contemporaneous returns.
\end{abstract}

Keywords: Bottom-up sentiment; market return predictability JEL classification: G12, G41

1 Financial support from the National Natural Science Foundation of China (No. 71571197), the 111 Project (No. B17050) and the Beijing Natural Science Foundation (No. 9152016) are gratefully acknowledged.

2 Jiaqi Guo, School of Economics, Finance and Accounting, Faculty of Business and Law, Coventry University, Coventry, CV1 5ED, UK. Tel: +44 (0) 247765 8410. Email: ac9510@coventry.ac.uk.

${ }^{3}$ Youwei Li, Hull University Business School, University of Hull, HU6 7RX, UK. Tel: +44 (0) 1482 462119. Email: Youwei.Li@hull.ac.uk.

${ }^{4}$ Min Zheng, Corresponding author, China Institute for Actuarial Science, Central University of Finance and Economics, 39 South College Road, Haidian District, Beijing, China, 100081. Email: mzheng@,cufe.edu.cn. 


\section{Introduction}

The role of investor sentiment in financial markets has been examined by extensive studies. For example, Brown and Cliff $(2004,2005)$ find that investor sentiment is positively correlated with market contemporaneous returns and negatively correlated with future market returns over the next 1 to 3 years. Baker and Wurgler (2007) find evidence on the sentiment-return relation at portfolio level and Baker et al. (2012) do not provide strong statistical evidence of sentiment in predicting future market returns in the regression analysis. Huang et al. (2015) adapt the Baker and Wurgler (2006) sentiment index and find greater predictive power for stock market returns. However, the literature still lacks sufficient evidence on the predictive power of sentiment of overall market returns at monthly frequency. Moreover, previous studies focus on the top-down approach of sentiment (e.g., the Baker and Wurgler (2006) sentiment index and the Investor Intelligence index (Brown and Cliff, 2004)). The top-down sentiment hardly takes account of every single stock's sentiment, so an individual stock sentiment may not be reflected in the top-down approach but can be captured by the bottom-up approach that uses many individual stocks' sentiment. For instance, investors may be on average optimistic about future market prospect, but they may be pessimistic about a specific stock. Therefore, such pessimism may be ignored by a market-wide sentiment measure and can be only identified from the bottom-up approach. Yu (2011) suggests that the bottom-up approach is better than the top-down approach since the signal-tonoise ratio is lower. Overall, the bottom-up market sentiment has some better properties than the top-down approach. In addition, Aboody et al. (2018) provide a robust measure of firm-specific sentiment, which enables us to create a better noise-free aggregate sentiment measure for the market under the "bottom-up" approach.

Motivated by those concerns, this paper investigates asset pricing implications of the bottom-up sentiment on the market portfolio. We construct a market sentiment measure using the bottom-up approach by aggregating firm-level sentiment and examine the relationship between the market sentiment and its subsequent returns. We argue that if 
the market is optimistic, stock prices would be driven away from its intrinsic value, resulting in high contemporaneous returns. This is also the source of low subsequent returns over long term as stock prices eventually revert to its fundamental value. Empirically, data support our hypothesized relationships.

\section{Data and Methodology}

This paper uses the Aboody et al.'s (2018) overnight return as a proxy for firm-specific investor sentiment. The data are provided by the CRSP database and are adjusted for stock splits, stock dividends and cash dividends. Only common stocks (SHRCO=10 or 11) listed on the NYSE/Amex/Nasdaq are included. Our sample period is from July 1992, which is the first month for opening stock price data available in CRSP, to December 2017. The overnight return of firm $i$ for day $d$ is measured as the difference between the stock opening price on day $d$ and the closing price on day $d-1$ divided by the closing price on day $d-1$. The overnight return for stock $i$ during month $t$ is defined as the average of the overnight returns with minimum 15 observations for that month multiplying 22 by assuming 22 trading days per month. The data of monthly market returns (NYSE/Amex/Nasdaq value-weighted index returns including distributions) are obtained from the CRSP.

The market sentiment, measured by the bottom-up approach, is the cross-sectional equally-weighted average of individual stock sentiment as follows:

$$
\text { Sent }_{t}=\frac{1}{n} \sum_{i=1}^{n} \text { Sent }_{i, t}
$$

where $\operatorname{Sent}_{i, t}$ is the sentiment of stock $i$ in month $t$ measured by overnight returns. Table 1 shows summary statistics of the bottom-up market sentiment and market returns with different horizons. The time-series mean of Sent $t_{t}$ is $-0.7 \%$ with a standard deviation of $3.2 \%$. The average of the market return $R_{t}^{M}$ is $0.9 \%$ per month with a standard deviation of $4.2 \%$ in our sample.

[Insert Table 1 here] 


\section{Empirical Results}

In this section, we investigate the association between the bottom-up sentiment and market returns in a multivariate regression setting as follows:

$$
R_{t, t+h}^{M}=\alpha+\beta * \text { Sent }_{t}+\sum \gamma_{i} * \text { Control }_{i, t}+\varepsilon_{t}
$$

where $R_{t, t+h}^{M}$ is the market cumulative return from months $t$ to $t+h$ and the range of $h$ is from 0 to 36 months. Sent $t$ is the equally-weighted market sentiment defined by (1) and Control is a vector of explanatory variables. The coefficient $\beta$ represents the sensitivity of market returns to the bottom-up market sentiment. We hypothesize excessive optimism drives market overvaluation resulting in subsequent corrections over the long run, which corresponds to a negative $\beta$, while current optimism would result in high contemporaneous returns corresponding to a positive $\beta$.

First, we estimate the regression without control variables. The results are shown in Panel A of Table 2. The coefficient of the sentiment on contemporaneous market returns is 0.457 , with a $t$-statistic of 5.67, which is consistent with the result of Brown and Cliff (2004) and implies that when the sentiment is high, investors should drive up prices, resulting in high contemporaneous returns. ${ }^{5}$ Columns 3 to 6 present the regression results for the future market returns from one month to three years on the bottom-up sentiment. The coefficients of the sentiment for all future return horizons are negative. For the case of the one-month horizon, consistent with the result of Brown and Cliff (2004), the coefficient of the sentiment is not significant, which implies the sentiment has weak explanatory power on the one-month's future market returns, suggesting that the sentiment is not a useful predictor for the subsequent near-term returns. It also indicates that the correction of mispricing does not occur immediately, so the significant reversal cannot be observed within a short period. However, from the six-month horizon, the coefficients of the sentiment become statistically significant. The effect of the

\footnotetext{
${ }^{5}$ We use the Newey and West (1987) standard errors to calculate the $t$-statistics, with the number of lags being equal to the return horizons.
} 
sentiment almost doubles going from 6-month to 1-year return horizons and increases further up to the 3-year horizon. ${ }^{6}$

[Insert Table 2 here]

Next, we investigate the predictive power of the sentiment when controlling for a set of potential variables that have impact on market returns. These variables are reviewed in Welch and Goyal (2008). They include price-earnings ratio (PE), consumption wealth ratio (CAY), dividend-price ratio (DP), treasury bills (TBL), long term yield on government bonds (LTY), the difference between long term and the Treasury-bill yields (TERMSPREAD), corporate bond returns (CORPRET), the default spread between BAA and AAA bond yields (DFSPREAD), and the lag rate of inflation (INFL). ${ }^{7}$ Here, the consumption wealth ratio is in a quarterly basis, so we transform the variable into a monthly basis using the last available values. The other variables are in a monthly basis.

We first estimate the predictive time-series regression by adding one control variable every time. The results are shown in Panel B of Table $2 .^{8}$ The sentiment is still a significant predictor of the future market returns at all 1-year to 3-year horizons while for 6-month horizon the sentiment is significant in most cases. For example, when we control PE, the coefficient of sentiment is -0.531 at 6 -month horizon and becomes 4.666 at 3-year horizon. Next, all control variables are included in the regression. The results are shown in Panel C of Table 2. The estimates of $\beta$ are generally consistent with those in Panel A of Table 2 without control variables. The sensitivity coefficient $\beta$ is negative across all future return horizons and is statistically significant at 6-month to 3year horizons. The longer return horizons, the more statistically significant of $\beta$, suggesting that the sentiment has more power to track long-term returns. Moreover, we compare the market return predictability of our bottom-up (Sent) and Baker and

\footnotetext{
6 In unreported results, the coefficient increases slightly from three-year to four-year horizons.

7 The data are obtained from Amit Goyal's website: http://www.hec.unil.ch/agoyal

${ }^{8}$ For brevity, only the coefficient and the statistical significance of sentiment are reported.
} 
Wurgler (2006; Sent_BW) sentiment measures. ${ }^{9}{ }^{10}$ The results are presented in Panel D of Table 2 . We observe that the bottom-up sentiment measure is positively correlated with contemporaneous market returns while the Baker and Wurgler's sentiment index is negatively correlated with them. Especially, only the coefficient of Sent on contemporaneous market returns is statistically significant. The ex post returns are negatively related to both Sent and Sent_BW. The two sentiment measures have similar explanatory power, suggesting they may capture different aspects of investor sentiment.

The findings of Table 2 are consistent with our hypothesis that when market is optimistic, the market valuation is higher than its fundamental value, resulting in subsequent low returns. Furthermore, the sensitivity coefficient $\beta$ is significantly positive for contemporaneous returns in all three panels. Nevertheless, the incremental effect of the bottom-up market sentiment does not disappear, suggesting the presence of a robust relation between the sentiment and future market returns.

\section{Conclusion}

This paper studies the asset pricing implication of the bottom-up sentiment for the market portfolio. We find the substantial evidence that the sentiment negatively predicts the future market returns over the long run. Contemporaneously, the variation of the sentiment is positively correlated with market returns.

\section{References}

Aboody, D., Even-Tov, O., Lehavy, R., Trueman, B., 2018. Overnight returns and firm-

\footnotetext{
${ }^{9}$ Baker and Wurgler's (2006) construct sentiment index as the first principle component of six proxies: the closed-end fund discount, the number of IPOs and first day returns of IPOs, NYSE turnover, the dividend premium and the equity share of total new issues. Their sentiment index also removes six economic fundamentals that are growth in industrial production, real growth in durable and nondurable consumptions, growth in services consumption, growth in employment and an NBER recession indicator. ${ }^{10}$ The correlation between the bottom-up and BW sentiment measures is 0.052 , which is significant at the $1 \%$ level. The low correlation suggests that they capture different aspects of investor sentiment.
} 
specific investor sentiment. J. Financ. Quant. Anal. 53 (2), 485-505.

Baker, M., Wurgler, J., 2006. Investor sentiment and the cross-section of stock returns. J. Financ. 61 (4), 1645-1680.

Baker, M., Wurgler, J., 2007. Investor sentiment in the stock market. J. Econ. Perspect. $21(2), 129-152$.

Baker, M., Wurgler, J., Yuan, Y., 2012. Global, local, and contagious investor sentiment. J. Financ. Econ. 104 (2), 272-287.

Brown, G., Cliff, M., 2004. Investor sentiment and the near-term stock market. J. Empir. Financ. $11(1), 1-27$.

Brown, G., Cliff, M., 2005. Investor sentiment and asset valuation. J. Bus. 78(2), 405440.

Huang, D., Jiang, F., Tu, J., Zhou, G., 2015. Investor sentiment aligned: a powerful predictor of stock returns. Rev. Financ. Stud. 28 (3), 791-837.

Newey, W., West, K., 1987. A simple, positive semi-definite, heteroskedasticity and autocorrelation consistent covariance matrix. Econometrica. 55, 703-708.

Welch, I., Goyal, A., 2008. A comprehensive look at the empirical performance of equity premium prediction. Rev. Financ. Stud. 21 (4), 1455-1508.

Yu, J., 2011. Disagreement and return predictability of stock portfolios. J. Financ. Econ. 99, 162-183. 


\section{Table 1 Summary Statistics}

Table 1 shows some summary statistics for the bottom-up sentiment and market portfolio returns with different return horizons. Sent $t_{t}$ is the equally weighted market sentiment index measured bottom-up using firm-specific sentiment in the market portfolio. $R_{t, t+h}^{M}$ is the CRSP value-weighted return (including distributions) from month $t$ to month $t+h$. For each variable, the sample period, the number of observations (N), the mean, standard deviation (STD), minimum (MIN), and maximum (MAX) are reported.

\begin{tabular}{lllllll}
\hline & Sample Period & N & MEAN & STD & MIN & MAX \\
\hline Sent $t_{t}$ & $1992.07-2017.12$ & 307 & -0.007 & 0.032 & -0.134 & 0.065 \\
$R_{t}^{M}$ & $1992.07-2017.12$ & 307 & 0.009 & 0.042 & -0.185 & 0.114 \\
$R_{t, t+6}^{M}$ & $1992.07-2017.12$ & 307 & 0.053 & 0.113 & -0.429 & 0.432 \\
$R_{t, t+12}^{M}$ & $1992.07-2017.12$ & 295 & 0.109 & 0.172 & -0.441 & 0.576 \\
$R_{t, t+24}^{M}$ & $1992.07-2016.12$ & 283 & 0.228 & 0.274 & -0.453 & 0.969 \\
$R_{t, t+36}^{M}$ & $1992.07-2015.12$ & 271 & 0.357 & 0.375 & -0.419 & 1.230 \\
\hline
\end{tabular}




\section{Table 2 Sentiment and Market Return}

Table 2 reports the regression results of market returns on the equally weighted sentiment. The sample period is from July 1992 to December 2017. Sent $t_{t}$ is the equally weighted market sentiment index measured by the bottom-up approach using firm-specific sentiment. Panel A shows the results regressing the market returns only on the equally-weighted sentiment index. In Panel B, the market returns not only depend on the sentiment index, but also rely on explanatory variables (as in Welch and Goyal (2008)), controlling for one explanatory variable every time. These explanatory variables include: price-earnings ratio $(\mathrm{PE})$, book to market ratio $(\mathrm{BM})$, consumption wealth ratio (CAY), dividend-price ratio (DP), treasury bills (TBL), long term yield on government bonds (LTY), the difference between long term and the Treasury-bill yields (TERMSPREAD), corporate bond returns (CORPRET), the default spread between BAA and AAA bond yields (DFSPREAD), and the lag rate of inflation (INFL). Panel C reports the regression results of the market returns on the sentiment, controlling all explanatory variables together. For brevity, only the coefficient of Sent $t_{t}$ is shown in Panels B and C. Sent_B $W_{t}$ is the Baker and Wurgler's (2006) monthly sentiment index. Panel D reports the regression results of market returns on Sent $t$ and Sent_BW $W_{t}$. The $t$-statistics are calculated using the method given by Newey and West (1987) with the number of lags being equal to the return horizons. *, ** and *** indicate statistical significance at the $10 \%, 5 \%$ and $1 \%$, respectively. 


\begin{tabular}{|c|c|c|c|c|c|c|}
\hline Return Horizon (in months) & 0 & 1 & 6 & 12 & 24 & 36 \\
\hline \multicolumn{7}{|c|}{ Panel A: market return on sentiment } \\
\hline Sent & $0.457 * * *$ & -0.089 & $-0.532 * *$ & $-1.052 * *$ & $-2.462 * *$ & $-4.800 * * *$ \\
\hline$t$-stat. & $(5.67)$ & $(-1.39)$ & $(-2.46)$ & $(-2.37)$ & $(-2.24)$ & $(-2.70)$ \\
\hline Constant & $0.012 * * *$ & $0.008 * * *$ & $0.049 * * *$ & $0.101^{* * *}$ & $0.207 * * *$ & $0.316^{* * *}$ \\
\hline$t$-stat. & $(5.54)$ & $(3.26)$ & $(3.62)$ & $(3.64)$ & $(3.60)$ & $(3.55)$ \\
\hline $\mathrm{R}^{2}$ & $11.95 \%$ & $0.45 \%$ & $2.24 \%$ & $3.86 \%$ & $8.39 \%$ & $17.66 \%$ \\
\hline \multicolumn{7}{|c|}{ Panel B: Coefficients of sentiment, controlling other variables one every time } \\
\hline $\mathrm{PE}$ & $0.459 * * *$ & -0.083 & $-0.531 * * *$ & $-1.022 * *$ & $-2.457 * *$ & $-4.666^{* * *}$ \\
\hline DP & $0.491^{* * *}$ & -0.045 & $-0.554^{* *}$ & $-1.047^{* *}$ & $-2.281 * *$ & $-4.142 * * *$ \\
\hline CAY & $0.629^{* * *}$ & -0.099 & -0.364 & $-0.629 *$ & $-1.322 *$ & $-2.484 * *$ \\
\hline TBL & $0.553^{* * *}$ & -0.097 & $-0.634 * * *$ & $-1.357 * * *$ & $-3.466^{* * *}$ & $-6.435 * * *$ \\
\hline LTY & $0.621^{* * *}$ & -0.126 & $-0.718^{* * *}$ & $-1.305^{* * *}$ & $-2.999 * * *$ & $-5.010^{* * *}$ \\
\hline CORPRET & $0.462 * * *$ & -0.084 & $-0.518^{* * *}$ & $-1.034 * * *$ & $-2.446^{* * *}$ & $-4.767 * * *$ \\
\hline DFSPREAD & $0.509^{* * *}$ & -0.075 & $-0.576^{* * *}$ & $-1.126^{* * *}$ & $-2.894 * * *$ & $-5.169 * * *$ \\
\hline TERMSPREAD & $0.458^{* * *}$ & -0.088 & $-0.532 * *$ & $-1.080^{* * *}$ & $-2.560 * *$ & $-4.997 * * *$ \\
\hline INFL & $0.456^{* * *}$ & -0.091 & $-0.521 * *$ & $-1.029 * *$ & $-2.431 * * *$ & $-4.783 * * *$ \\
\hline \multicolumn{7}{|c|}{ Panel C: Coefficients of sentiment, controlling all explanatory variables together } \\
\hline Sent & $0.671^{* * *}$ & -0.115 & $-0.439^{*}$ & $-0.663^{*}$ & $-1.672 * * *$ & $-2.286^{* *}$ \\
\hline$t$-stat. & $(6.47)$ & $(-1.31)$ & $(-1.81)$ & $(-1.91)$ & $(-3.28)$ & $(-2.54)$ \\
\hline All other variables & Yes & Yes & Yes & Yes & Yes & Yes \\
\hline $\mathrm{R}^{2}$ & $21.73 \%$ & $3.31 \%$ & $12.41 \%$ & $26.11 \%$ & $45.17 \%$ & $66.40 \%$ \\
\hline \multicolumn{7}{|c|}{ Panel D: Coefficients of bottom-up and Baker and Wurgler (2006) sentiment measures } \\
\hline Sent & $0.441 * * *$ & $-0.158 * *$ & $-0.964 * * *$ & $-1.897 * * *$ & $-3.683 * * *$ & $-5.930 * * *$ \\
\hline$t$-stat. & $(4.93)$ & $(-2.19)$ & $(-5.32)$ & $(-4.83)$ & $(-3.61)$ & $(-3.79)$ \\
\hline Sent_BW & -0.002 & $-0.011^{*}$ & $-0.067 * * *$ & $-0.134 * * *$ & $-0.208 * * *$ & $-0.194 * * *$ \\
\hline$t$-stat. & $(-0.41)$ & $(-1.92)$ & $(-4.46)$ & $(-6.10)$ & $(-5.56)$ & $(-4.05)$ \\
\hline
\end{tabular}

